\title{
Inflammation and Atherosclerosis: Evolving Concepts Leading the Development of New Therapies
}

\section{Alessandro Mauriello ${ }^{1 *}$ and Giuseppe Sangiorgi ${ }^{2}$}

${ }^{1}$ Department of Surgical Pathology, University of Rome Tor Vergata, Italy

${ }^{2}$ Department of Cardiology, University of Rome Tor Vergata, Italy

\section{Editorial}

Several anatomic and clinical studies have demonstrated that acute myocardial infarction (AMI) and cerebrovascular accidents are due to thrombosis and rupture of a vulnerable atherosclerotic plaque, rather than to the degree of stenosis [1-3]. Many efforts have been recently made using non-invasive imaging techniques [4-7] for identifying plaques at high risk of disruption leading to thrombosis, generally defined as "vulnerable plaques" $[8,9]$. Unlike the stable plaque that shows a chronic inflammatory infiltrate, the vulnerable and ruptured plaque is characterized by a chronic "active" inflammation. The "active" inflammation is mainly represented by T-lymphocytes and macrophages which are activated towards a pathway of inflammatory response and secrete cytokines and lytic enzymes which in turn cause fibrous cap thinning predisposing the plaque to rupture [10-13]. Moreover, some pathologic, clinical and angiographic observations seem to suggest the possibility that the principal cause of coronary instability is not to be found in the vulnerability of a single atherosclerotic plaque, but in the presence of multiple vulnerable plaques in the entire coronary tree [12], associated with a widespread inflammatory process consisting of macrophagic cells and activated T lymphocytes [11].

The adaptive immune response has a crucial role in determining the progression and regression of the atherosclerotic disease. A critical role in leading both an immune pro-atherogenic- and a protective response is played by $\mathrm{T}$ CD4+ lymphocytes sub-population. The selective recruitment of a specific subtype of $\mathrm{CD} 4+$ cells plays a major role in the transformation of a stable plaque in a vulnerable or ruptured one $[10,14]$. Past studies have described two subtypes of CD4+ cells with a juxtaposed role, namely Th1 and Th2 cells [15]. Th1 cells secreting pro-inflammatory cytokines, such as IFNg, promote macrophage activation, inflammation, and atherosclerosis [16], whereas Th2 cells (cytokine pattern IL-4, IL-5 and IL-10) mediate antibody production and generally have anti-inflammatory and anti-atherogenic effects [17]. Later, a third subset of CD4+ cells, called Th17, producing IL-17, has been described $[18,19]$. While the function of these cell subtypes is not completely elucidated, experimental studies suggest that Th17 cells are highly pro-inflammatory and may play an important role in the autoimmunity and in host defense against extracellular pathogens, which are not efficiently cleared by Th1-type and Th2-type immunity. Indirect evidences however suggest a possible involvement for such effector $\mathrm{T}$ cell subset in the acute cardiovascular diseases. Patients with AMI and unstable angina show an increased amount of Th17 cell, Th17 related factors such as IL-17, IL-6, IL-23 [20]. Recently, the role of regulatory T cells (Tregs), a sub-population of CD4+ Tcells, in atherosclerosis, has been also pointed out. Tregs play a central role in inducing and maintaining immunologic tolerance [14]. Deficiency or dysfunction of these cells lead to autoimmunity or aggravated pathogen-induced inflammation [14]. An important population of Tregs is formed by the CD4+CD25+ regulatory $\mathrm{T}$ cells which express the transcription factor FOXP3 (Forkhead Box Protein P3) [21]. It was shown that the amount of Tregs is decreased in the peripheral blood of patients with acute coronary syndromes [20]. However, the in situ presence of Th17 and Tregs in human atherosclerotic plaques has not been well investigated and the function of novel subsets of T cells (Tregs and Th17) in plaque progression remains largely unknown. Also the role of the humoral response in the development of atherosclerosis remains to be clarified. However, it has been hypothesized that Th1type immune responses promote disease, whereas humoral immunity has protective effects [22]. This hypothesis is supported primarily by the observation that treatment with anti oxLDL inhibits atherosclerosis in experimental models [22]. A reduction of size of atherosclerotic lesions has been observed also with anti CD20 antibody in mice. Studies in humans are scarce and contradictory.

Despite the significant increase in our knowledge of the role of inflammation in the atherosclerosis there are a number of issues to be solved.

First, several studies have demonstrated that inflammation plays a key role in the inception, progression and destabilization of atherosclerotic plaques and in the occurrence of acute clinical syndromes. However, recent observations, mostly experimental, hypothesize that the activation of the immune response not only promotes disease (adaptive immunity) but has also protective effects (humoral immunity).

Second, it can be hypothesized that, regardless of the type of causative agent, the changes in the adaptive immune response play a fundamental role in atherosclerotic disease progression and regression. In this setting, data available in literature are incomplete. T-cells are not only the major effectors of cell mediated immunity, but are obviously involved in antibody responses and therefore require deeper insight. A very important role, not yet well studied, is that of dendritic cells, namely cells specialized in antigen presentation with a key role in the induction of primary immune response and in the regulation of $\mathrm{T}$ lymphocytes differentiation, as well as in mechanisms of central and peripheral tolerance aiming at the elimination of T lymphocytes that are potentially self-reactive towards self-antigens [23]. In this contest also the pro-inflammatory functions of platelets on atherosclerosis need to be better outlined. Platelets are able to interact with a large variety of cell types implicated in the pathophysiology of vascular inflammation and to transport and synthesize cytokines, chemokines, and lipid mediators, thereby favoring the development of atherosclerotic disease.

Third, the role of M1 and M2 macrophage subpopulations in various states of evolution of atherosclerotic plaque is not yet been completely clarified [24]. Investigators have hypothesized that monocytes will differentiate into specific macrophage subpopulations

*Corresponding author: Alessandro Mauriello, Cattedra di Anatomia ed Istologia Patologica, Dipartimento di Biomedicina e Prevenzione, University of Rome "Tor Vergata”, Via Montpellier 1, 00133 Roma, Italy, Tel: +39 - 06 - 2023751; Fax: +3906-20902209; E-mail: alessandro.mauriello@uniroma2.it

Received September 27, 2012; Accepted September 27, 2012; Published September 30, 2012

Citation: Mauriello A, Sangiorgi G (2012) Inflammation and Atherosclerosis: Evolving Concepts Leading the Development of New Therapies. J Metabolic Synd 1:e106. doi:10.4172/2167-0943.1000e106

Copyright: (c) 2012 Mauriello A, et al. This is an open-access article distributed under the terms of the Creative Commons Attribution License, which permits unrestricted use, distribution, and reproduction in any medium, provided the original author and source are credited. 
Citation: Mauriello A, Sangiorgi G (2012) Inflammation and Atherosclerosis: Evolving Concepts Leading the Development of New Therapies. J Metabolic Synd 1:e106. doi:10.4172/2167-0943.1000e106

Page 2 of 2

in response to alternative cytokine environments [25,26]. Classic M1-activated macrophages are part of polarized Th1 responses and are known to play a pivotal role in the progression of atherosclerosis. Alternative macrophage activation (M2), originally discovered as a response to IL-4, is oriented to the promotion of tissue remodeling and angiogenesis [25]. Patients with coronary artery disease have higher percentages of M1 monocytes than healthy cohorts, while normal coronary intimal vasculature predominantly contains M2 resembling macrophages [24-26]. The question that still remains to be answered is: are all monocytes recruited, or do subsets with specific functions exist, and does recruitment depend on the stage of the lesions?

Fourth, the role of humoral immune response in atherogenesis remains to be clarified. Although some clinical and epidemiological studies have found positive correlations between the presence of antibodies specific intraplaque antigens such as oxLDL and the progression of atherosclerosis, other studies have not detected any correlation. However, it was assumed that the humoral immunity has protective effects, likely through the elimination of antigens before they reach plaques, as demonstrated by several studies showing that splenectomy increases atherosclerosis in Apoe-/- mice. This hypothesis is supported by the observation that treatment with oxLDL antibodies inhibits atherosclerosis in experimental models. Similarly, IL-2/antiIL-2 mAb treatment in vivo attenuates atherosclerosis via selective Tregs expansion [27].

Recent advances in understanding the immune and humoral responses involved in the various evolutionary phases of atherosclerotic disease may better clarify the pathogenetic links between plaque inflammation and atherosclerosis progression and regression, and to identify new possible targets for atherosclerotic plaque stabilization. Recent trials using novel therapeutic approaches aimed at modulating plaque inflammation, such as Interleukin- $1 \beta$ inhibition [28], support the idea that modulation of immune responses may reduce inflammatory damage and enhance plaque healing and stabilization.

\section{References}

1. Spagnoli LG, Mauriello A, Sangiorgi G, Fratoni S, Bonanno E, et al. (2004) Extracranial thrombotically active carotid plaque as a risk factor for ischemic stroke. JAMA 292: 1845-1852.

2. Fuster V, Badimon L, Badimon JJ, Chesebro JH (1992) The pathogenesis of coronary artery disease and the acute coronary syndromes (1). N Engl J Med 326: 242-250.

3. Ambrose JA, Tannenbaum MA, Alexopoulos D, Hjemdahl-Monsen CE, Leavy $\mathrm{J}$, et al. (1988) Angiographic progression of coronary artery disease and the development of myocardial infarction. J Am Coll Cardiol 12: 56-62.

4. Fleg JL, Stone GW, Fayad ZA, Granada JF, Hatsukami TS, et al. (2012) Detection of High-Risk Atherosclerotic Plaque: Report of the NHLBI Working Group on Current Status and Future Directions. JACC Cardiovasc Imaging 5: 941-955

5. Puri R, Worthley MI, Nicholls SJ (2011) Intravascular imaging of vulnerable coronary plaque: current and future concepts. Nat Rev Cardiol 8: 131-139.

6. Fabiano S, Mancino S, Stefanini M, Chiocchi M, Mauriello A, et al. (2008) High-resolution multicontrast-weighted MR imaging from human carotid endarterectomy specimens to assess carotid plaque components. Eur Radiol 18: $2912-2921$

7. Coli S, Magnoni M, Sangiorgi G, Marrocco-Trischitta MM, Melisurgo G, et al. (2008) Contrast-enhanced ultrasound imaging of intraplaque neovascularization in carotid arteries: correlation with histology and plaque echogenicity. J Am Coll Cardiol 52: 223-230

8. Burke AP, Farb A, Malcom GT, Liang YH, Smialek J, et al. (1997) Coronary risk factors and plaque morphology in men with coronary disease who died suddenly. N Engl J Med 336: 1276-1282.

9. Virmani R, Kolodgie FD, Burke AP, Farb A, Schwartz SM (2000) Lessons from sudden coronary death: a comprehensive morphological classification scheme for atherosclerotic lesions. Arterioscler Thromb Vasc Biol 20: 1262-1275.

10. Hansson GK, Hermansson A (2011) The immune system in atherosclerosis. Nat Immunol 12: 204-212.

11. Spagnoli LG, Bonanno E, Mauriello A, Palmieri G, Partenzi A, et al. (2002) Multicentric inflammation in epicardial coronary arteries of patients dying of acute myocardial infarction. J Am Coll Cardiol 40: 1579-1588.

12. Mauriello A, Sangiorgi G, Fratoni S, Palmieri G, Bonanno E, et al. (2005) Diffuse and active inflammation occurs in both vulnerable and stable plaques of the entire coronary tree: a histopathologic study of patients dying of acute myocardial infarction. J Am Coll Cardiol 45: 1585-1593.

13. Spagnoli LG, Bonanno E, Sangiorgi G, Mauriello A (2007) Role of inflammation in atherosclerosis. J Nucl Med 48: 1800-1815.

14. Mallat Z, Ait-Oufella H, Tedgui A (2007) Regulatory T-cell immunity in atherosclerosis. Trends Cardiovasc Med 17: 113-118.

15. Daugherty A, Rateri DL (2002) T lymphocytes in atherosclerosis: the yin-yang of Th1 and Th2 influence on lesion formation. Circ Res 90: 1039-1040.

16. Benagiano M, Azzurri A, Ciervo A, Amedei A, Tamburini C, et al. (2003) Thelper type 1 lymphocytes drive inflammation in human atherosclerotic lesions. Proc Natl Acad Sci U S A 100: 6658-6663.

17. Szodoray P, Timar O, Veres K, Der H, Szomjak E, et al. (2006) TH1/TH2 imbalance, measured by circulating and intracytoplasmic inflammatory cytokines--immunological alterations in acute coronary syndrome and stable coronary artery disease. Scand J Immunol 64: 336-344

18. Bettelli E, Korn T, Kuchroo VK (2007) Th17: the third member of the effector T cell trilogy. Curr Opin Immunol 19: 652-657.

19. Chen Z, O'Shea JJ (2008) Th17 cells: a new fate for differentiating helper cells. Immunol Res 41: 87-102.

20. Cheng X, Yu X, Ding YJ, Fu QQ, Xie JJ, et al. (2008) The Th17/Treg imbalance in patients with acute coronary syndrome. Clin Immunol 127: 89-97.

21. George $\mathrm{J}$ (2008) Mechanisms of disease: the evolving role of regulatory T cells in atherosclerosis. Nat Clin Pract Cardiovasc Med 5: 531-540.

22. Shah PK, Chyu KY, Fredrikson GN, Nilsson J (2005) Immunomodulation of atherosclerosis with a vaccine. Nat Clin Pract Cardiovasc Med 2: 639-646.

23. Koltsova EK, Garcia Z, Chodaczek G, Landau M, McArdle S, et al. (2012) Dynamic T cell-APC interactions sustain chronic inflammation in atherosclerosis. J Clin Invest 122: 3114-3126

24. Mantovani A, Garlanda C, Locati M (2009) Macrophage diversity and polarization in atherosclerosis: a question of balance. Arterioscler Thromb Vasc Biol 29: 1419-1423.

25. Johnson JL, Newby AC (2009) Macrophage heterogeneity in atherosclerotic plaques. Curr Opin Lipidol 20: 370-378.

26. Woollard KJ, Geissmann F (2010) Monocytes in atherosclerosis: subsets and functions. Nat Rev Cardiol 7: 77-86.

27. Dinh TN, Kyaw TS, Kanellakis P, To K, Tipping P, et al. (2012) Cytokine Therapy With Interleukin-2/Anti-Interleukin-2 Monoclonal Antibody Complexes Expands CD4+CD25+Foxp3+ Regulatory T Cells and Attenuates Development and Progression of Atherosclerosis. Circulation 126: 1256-1266.

28. Ridker PM, Thuren T, Zalewski A, Libby P (2011) Interleukin-1î inhibition and the prevention of recurrent cardiovascular events: rationale and design of the Canakinumab Anti-inflammatory Thrombosis Outcomes Study (CANTOS). Am Heart J 162: 597-605. 